\title{
Reflets
}

Revue ontaroise d'intervention sociale et communautaire

\section{L'exclusion : pistes de réflexion en contextes minoritaires}

\section{Madeleine Dubois et Michèle Kérisit}

Volume 11, numéro 1, 2005

Exclusion sociale

URI : https://id.erudit.org/iderudit/013057ar

DOI : https://doi.org/10.7202/013057ar

Aller au sommaire du numéro

Éditeur(s)

Reflets : Revue ontaroise d'intervention sociale et communautaire

ISSN

1203-4576 (imprimé)

1712-8498 (numérique)

Découvrir la revue

Citer ce document

Dubois, M. \& Kérisit, M. (2005). L’exclusion : pistes de réflexion en contextes minoritaires. Reflets, 11(1), 10-18. https://doi.org/10.7202/013057ar

Tous droits réservés (C) Reflets : Revue ontaroise d'intervention sociale et communautaire, 2005
Ce document est protégé par la loi sur le droit d'auteur. L'utilisation des services d'Érudit (y compris la reproduction) est assujettie à sa politique d'utilisation que vous pouvez consulter en ligne.

https://apropos.erudit.org/fr/usagers/politique-dutilisation/ 


\section{L'exclusion : pistes de réflexion en contextes minoritaires}

\section{Madeleine Dubois et Michèle Kérisit}

Intégré au vocabulaire des sciences sociales depuis une quinzaine d'années, le concept d'exclusion est investi de significations diverses, associées tant à la pauvreté, à certaines formes plus marquées de chômage, à la discrimination et au racisme, qu'aux phénomènes de rejet social qui marquent certains rapports entre groupes. Bien que critiquée pour son caractère vague et mouvant et objet de nombreux débats dans diverses disciplines des sciences sociales, l'exclusion demeure un concept important pour baliser la réflexion sur les liens qui caractérisent une société, les rapports de pouvoir et les processus de marginalisation qui s'y développent.

La présentation d'un numéro de Reflets sur le thème de l'exclusion appelle d'abord à une analyse plus approfondie des concepts qui y sont étroitement associés. Dans un numéro de la revue Lien social et politique de 1995 ayant pour thème "Y a-t-il des exclus? ", Robert Castel déplore l'usage non qualifié du terme et suggère certains éléments pour mieux cerner les caractéristiques qu'il recèle. Il souligne ainsi l'importance de nommer en quoi consiste le manque ou les manques auxquels se heurtent les personnes qu'on nomme des exclus afin d'en comprendre les origines. Cette mise en garde demeure pertinente et d'actualité.

Le sens qu'on accorde au terme d'exclusion se construit autour de deux dimensions principales qui seront accentuées en fonction des analyses proposées par les auteurs qui l'ont utilisé. Ces deux dimensions sont, d'une part, la participation, ou la non-participation, au marché du travail et, d'autre part, le fait 
de participer ou non à des réseaux sociaux (familiaux, mais aussi communautaires ou même politiques).

Par exemple, les recherches de Paugam (1993) sur la pauvreté et sur les personnes recevant des prestations d'aide sociale reposent sur une vision de la pauvreté comme "produit d'une construction sociale ». Selon cette vision, l'exclusion est avant tout liée à l'intégration dans les multiples niveaux de participation au monde du travail. La façon de cibler les individus pour les intégrer au travail, à travers des dispositifs d'aide, est donc essentielle pour comprendre les effets de l'exclusion qui se situe sur le plan de la "disqualification ». Des études des rapports entre les individus et les institutions d'assistance en France l'amènent ainsi à suggérer un modèle de disqualification comportant trois catégories, chacune représentant un type idéal qu'il nomme les "fragilisés ", les " assistés » et les "marginaux ». L'accent mis sur la création d'exclus à travers leurs rapports avec les institutions comporte cependant un risque : celui de catégoriser, voire d'étiqueter, les personnes comme ayant peu de capacité d'agir sur leur condition.

D'un autre côté, selon la perspective avancée par de Gaulejac et Leonetti dans La lutte des places, l'exclusion est associée à un processus de désinsertion vécu selon des trajectoires de vie individuelles et profondément marquées par la manière dont les abordent les personnes qui y sont confrontées.Ainsi, la désinsertion se structure autour de deux axes, le premier comprenant les multiples lieux où se situent les ruptures (travail, famille) et le second se situant sur le plan des réactions face à ces dernières, influencées notamment par la valeur symbolique qu'elles accordent à ces ruptures. Moins marquée par un déterminisme social et institutionnel, l'expérience de l'exclusion s'inscrit donc dans la recherche d'une reconnaissance sociale, d'une dignité qui serait inhérente à la personne humaine, laissant une place à la subjectivité et à la possibilité de révolte contre l'exclusion.

Castel propose aussi une approche de l'exclusion axée sur un processus comportant divers éléments se structurant autour d'une " dégradation par rapport à une situation antérieure ". Par contre, pour comprendre le phénomène et sa portée, il importe selon lui, non pas de se limiter à décrire des " états de dépossession ", mais 
plutôt de "reconstruire le continuum des positions qui relient les in et les out, et de ressaisir la logique à partir de laquelle les in produisent des out " (1995:15).

Quoique s'articulant toutes deux autour de processus de démaillage ou de désarticulation sociale, l'approche de de Gaulejac et Leonetti s'intéresse davantage aux points de rupture, à leurs impacts sur la personne et au sens qu'elle leur accorde, alors que l'approche soutenue par Castel situe l'exclusion dans un contexte plus global, qui tient compte du rôle d'une variété d'acteurs, de systèmes et d'influences. Comme " le sort des exclus se joue pour l'essentiel avant qu'ils ne basculent » (1995:16), Castel met l'accent sur l'importance de nommer les conjonctures économiques et sociales qui contribuent aux étapes de la désaffiliation et de travailler à la création de politiques sociales préventives plutôt que d'investir toutes les ressources dans la réinsertion par le biais de la prise en charge de groupes ciblés et spécifiques. Sans pour autant mettre de côté les mécanismes visant à " réinsérer ", la lutte doit se concrétiser par un ensemble de mesures agissant sur divers plans pour mettre un frein au processus de désinsertion au travail ainsi qu'à la vulnérabilité des liens sociaux dans les réseaux de relations familiales et sociales.

Développée depuis plus de dix ans, l'analyse de l'exclusion en tant que processus de désaffiliation proposée par Castel demeure tout aussi pertinente aujourd'hui. Les malaises provoqués par les évolutions dans le monde du travail, de plus en plus imputables aux effets de la mondialisation qui s'accélère, amènent certains auteurs à introduire, au vocable de l'exclusion, le phénomène de "désocialisation". Ainsi, dans un article intitulé "Les nouvelles inégalités ", Bensaïd et al. soutiennent que "les nouvelles inégalités et la frustration qu'elles déclenchent trouvent leur source dans le malaise nouveau d'une civilisation postindustrielle, d'un monde du travail qui se précarise, d'une identité personnelle qui fait décliner sur un mode individuel les difficultés d'insertion dans une société qui se dérobe sans cesse à ses membres " (2004 :35). L'injonction de la réussite sociale, contrecarrée par les diverses délocalisations, la multiplication des emplois précaires et mal payés et les difficultés à trouver un équilibre dans les liens intimes et sociaux font que 
de plus en plus de personnes se sentent marginalisées, fragilisées et vulnérables, incapables de se sentir actrices à part entière de la direction de leur vie. L'exclusion qui, autrefois, désignait des processus de grande marginalisation dus à des problématiques comme l'alcoolisme, la toxicomanie, l'errance, se rapproche de plus en plus des centres de la vie sociale et c'est maintenant la personne ordinaire qui peut se sentir atteinte et fragilisée.

\section{Présentation des articles du numéro}

Même s'ils ne puisent pas nécessairement dans les fondements théoriques présentés ci-dessus, plusieurs des articles du présent numéro situent dans un contexte concret certains des facteurs conduisant des groupes ou des personnes à des situations de vulnérabilité ou de précarité pouvant mener à l'exclusion sociale. De fait, nous y trouvons deux perspectives sous lesquelles ont été examinés certains phénomènes d'exclusion : le rôle direct ou indirect de l'État dans l'accentuation des processus d'exclusion et la situation particulière où se trouvent certains groupes sociaux particulièrement vulnérables, en fonction de leur sexe, de leur âge ou de leur origine. À cela s'ajoute un appel à la création de solutions et d'un discours cohérent en Ontario français, en particulier dans le domaine de la santé, afin de créer des conditions favorisant la mise sur pied de services inclusifs.

Il est bien connu que le contexte minoritaire des francophones de l'Ontario les rend plus vulnérables à la perte de certaines ressources ou de certains services imposée par des politiques étatiques peu sensibles aux particularités de leurs communautés. L'étude de cas présentée par Madibbo et Labrie analyse comment deux organismes de Toronto offrant des services aux nouveaux arrivants francophones de cette ville ont été touchés par des politiques privilégiant certains mécanismes de financement au détriment d'autres, au lieu de se centrer sur une vision globale de la communauté et de ses besoins et particularités. L'analyse du discours produit dans le cadre d'entrevues avec divers acteurs 
sociaux, à la fois intervenants et participants dans les programmes offerts par les organismes, illustre les situations paradoxales résultant de la mise en place, par les bailleurs de fonds, de critères de financement étroitement définis. Ce faisant, l'État oblige les organismes à cibler certaines problématiques à l'exclusion de l'ensemble des facteurs de vulnérabilité, variés et complexes, qui affectent les personnes. Sont ainsi créées des filières d'exclusion au sein même d'organismes se voulant des lieux d'inclusion et d'intégration sociale.

Les formes de l'exclusion sont évidemment marquées par le contexte historique, politique et culturel dans lequel elles émergent. L'article de Lassaad Labidi sur la condition des retraités en Tunisie montre comment l'absence de politiques étatiques et les aspects culturels et symboliques entourant le vieillissement interagissent pour exclure les personnes âgées de la vie sociale et économique dans leur pays.Ainsi, une loi interdisant le travail après la retraite, combinée à l'absence de politiques visant la préparation à la retraite et le soutien aux personnes retraitées, se conjugue aux mutations dans l'organisation sociale traditionnelle du pays pour engendrer précarité, voire pauvreté, crises d'identité et sentiments de disqualification et d'exclusion chez les personnes de plus de 60 ans, âge obligatoire de la retraite dans ce pays.

L'âge n'est par ailleurs pas le seul facteur de vulnérabilité. Au Canada, les statistiques sur la pauvreté font largement état de la vulnérabilité des femmes. Trois articles de ce numéro de Reflets portent sur cette problématique avec, pour chacun, un angle différent d'approche.

Dans sa réflexion sur le développement des services en matière de violence en Ontario français, Garceau nomme les facteurs qui rendent particulièrement vulnérables les femmes francophones de l'Ontario exposées à des situations de violence. Des conditions de vie particulières telles que la dispersion géographique, les lacunes au niveau du transport en commun en milieux ruraux et l'absence de services en français viennent s'ajouter aux compressions de l'État en matière d'aide sociale et de logement. Cette combinaison de facteurs a pour effet de brimer le pouvoir d'agir des femmes. Alors qu'on pourrait s'attendre à ce que le financement accordé 
tienne compte des contraintes particulières afin d'offrir un soutien adéquat aux femmes, les services en français sont au contraire sous-financés, accusant un décalage par rapport aux services en anglais.

Ces constatations quant à la quasi-inexistence de services en français s'adressant aux femmes victimes de violence trouvent écho dans l'article de Plante et ses collaboratrices qui ont étudié l'impact de l'expérience des conflits armés sur l'intégration de femmes francophones immigrantes et réfugiées en Ontario. Les auteures présentent les recommandations émergeant de cette étude, marrainée par Le mouvement des femmes immigrantes francophones. Non seulement les femmes qui ont traversé les atrocités de la guerre ne peuvent-elles pas compter sur un appui en français dans des services qui allégeraient leur souffrance, mais elles sont de plus confrontées à des mesures qui constituent autant de barrières légales et financières à leur intégration et à leur participation en tant que citoyennes.

La situation de ces femmes est d'ailleurs confirmée par l'étude du Conseil de planification sociale d'Ottawa examinant les revenus, l'éducation, l'accès au logement et à l'emploi pour les francophones d'Ottawa, hommes ou femmes. Présentée par MarieJosée Legault, cette étude met en lumière les difficultés auxquelles sont confrontées les personnes immigrantes, particulièrement les personnes de couleur, notamment sur le plan de l'accès à l'emploi, et ce, en dépit de niveaux d'études souvent plus élevés que dans l'ensemble de la communauté francophone.

L'article de Bruckert et de Parent sur les travailleuses de sexe offre une perspective différente en présentant une analyse de filières de marginalisation et d'exclusion résultant non seulement de politiques et de pressions sociales exercées par les diverses institutions sociales, mais aussi de formes de hiérarchisation internes accentuant l'exclusion au sein même des groupes de femmes impliquées dans les diverses formes de travail de sexe. L'analyse des entrevues menées par ces chercheurs auprès de travailleuses du sexe dans la région d'Ottawa présente aussi des situations paradoxales dans lesquelles se retrouvent ces personnes. Elles sont à la fois stigmatisées par leur travail et revendicatrices de 
conditions leur offrant certaines protections et la reconnaissance de leurs droits comme travailleuses.

Comme le disent Parent et Bruckert, les femmes qu'elles ont interviewées pour leur étude ne sont pas victimes passives de leur situation. De la même façon, les femmes survivantes de conflits armés et les personnes immigrantes et réfugiées expriment haut et clair ce qui ne va pas et ce qui devrait être fait. Ces personnes agissent aussi pour contrer leur situation et pour redéfinir leur condition à travers la parole et l'action. Cet aspect des phénomènes d'exclusion - la parole des personnes exclues — est souvent passé sous silence. C'est justement sur l'importance de donner la parole à un groupe de jeunes susceptibles d'être relégués aux marges de la société que porte l'article de Byrne et Lemay. Celui-ci traite d'une recherche effectuée auprès de jeunes francophones ayant vécu une partie de leur enfance ou de leur adolescence sous la tutelle d'une société de l'aide à l'enfance. Le but de la recherche est d'inviter les jeunes à discuter de leurs perceptions, de leurs expériences et de leurs attentes face aux personnes responsables de leur bien-être, plus particulièrement les travailleurs sociaux qui les ont accompagnés et soutenus et les familles d'accueil dans lesquelles ils ont habité.

Dans un numéro consacré au thème de l'exclusion, le rôle et la place des institutions sont maintes fois évoqués. Ainsi, certains articles examinent la façon dont les communautés de langue française en Ontario tentent de redéfinir les paramètres dans lesquels s'inscrivent leurs institutions afin d'apporter des solutions à une situation minorisante et excluante.

La santé constitue de toute évidence un des domaines où surgissent inégalités et difficultés de participation sociale. Dans leur analyse des discours sur la santé qu'ont tenus les organismes francoontariens jusqu'à la fin des années 90, Gilbert, Kérisit, Dallaire, Coderre et Harvey mettent en lumière les diverses revendications faites pour la mise en place et le développement des services de santé en français. Depuis le rapport Dubois de 1976, en passant par les discours ayant légitimé la création de centres de santé communautaire francophones entre 1988 et 1995, jusqu'à ceux ayant marqué la lutte pour la sauvegarde de l'Hôpital Montfort 
à la fin des années 1990, ces auteurs démontrent comment ces revendications sont redevables "d'un contexte sociopolitique contingent qui canalise les possibilités d'expression, tout en générant des espaces de création, par les interstices desquels s'expriment les visions du monde, les revendications collectives, les projets de société ". Cette analyse laisse entrevoir comment se profilent, dans les divers discours pour la revendication des droits, "des rapports de pouvoir et des luttes d'influence pour articuler des visions parfois bien différentes sur la santé ».Tout en notant l'absence d'un discours unitaire sur la santé, ils montrent l'importance accordée au contexte politique et à l'intervention de l'État (provincial ou fédéral) dans les discours portant sur la santé en français, plutôt qu'à la mobilisation et la participation populaire.

L'un des moyens que se sont donnés les francophones vivant en situation minoritaire au Canada afin de pouvoir revendiquer des services en français est le Programme de recherche national sur la santé des communautés francophones minoritaires présenté par Francine Desbiens. L'auteure met en lumière la pénurie d'études et de recherches sur la spécificité de la santé de ces communautés et sur l'absence de financement et de ressources pour faciliter la production de données probantes, mais aussi les premières initiatives prises par le Consortium national de formation en santé pour combler les lacunes.

\section{Conclusion}

Au terme de cette recension des articles reçus par Reflets pour son numéro sur l'exclusion que peut-on conclure?

En premier lieu, l'État est perçu comme un acteur particulièrement important dans la création de filières d'exclusion ou d'inclusion. Bien qu'en déclin, les filets de sécurité offerts par l'État jouent encore un rôle dans la capacité des communautés et des personnes de sortir de l'exclusion. Encore faut-il que cet État veuille et puisse jouer son rôle : tel n'est pas le cas dans 
certaines circonstances et dans certaines sociétés comme la société tunisienne. Encore faut-il qu'il soit attentif à l'ensemble des facteurs de vulnérabilité menant à l'exclusion. En effet, si l'accès à l'emploi et à l'employabilité est très important pour contrer la disqualification, si l'accès à des services de santé est crucial pour les personnes vulnérables, encore faut-il que les programmes et services toujours soutenus par l'État prennent en considération les autres dimensions de l'exclusion : désaffiliation et isolement, pauvreté et besoin d'appartenance. En somme, les dimensions sociales et socialisantes des services sont importantes afin de permettre un point d'ancrage dans la collectivité. Cela est particulièrement important pour le " filet de sécurité » francophone dont les mailles sont déjà très lâches, sinon trouées.

En second lieu, il est important de rappeler que les collectivités francophones, y compris les communautés de pratique représentées par les intervenants travaillant dans les services, jouent un rôle important dans la définition des problèmes et de leurs solutions. Cela ne signifie pas que toutes et tous doivent marcher d'un même pas et il est certain que des désaccords sur telle ou telle source d'exclusion et sur les solutions pour y remédier peuvent exister. Il est même sain qu'à l'intérieur des communautés francophones, un débat ait lieu et que de ces délibérations, l'on sorte renforcé dans son sentiment d'appartenir à une collectivité et donc d'être « inclus». Le courage de nommer l'exclusion fait partie des stratégies menant à l'inclusion.

\section{Bibliographie}

BENSAÏD, Jean, Daniel Cohen, Éric Maurin, Olivier Mongin (2004). «Les nouvelles inégalités », Esprit, février.

CASTEL, Robert (1995). "Les pièges de l'exclusion ", Lien social et politiques - RIAC, 34 : 74.

De GAULEJAC,Vincent et Isabel Taboada Léonetti (1994). La lutte des places, Marseille, Hommes et Perspectives.

PAUGAM, Serge (1993). La société française et ses pauvres, Paris, PUF. 